\title{
A new type of generalized quasi-Einstein manifold
}

Brijesh Gupta and Braj Bhushan Chaturvedi

Department of Pure \& Applied Mathematics Guru Ghasidas Vishwavidyalaya Bilaspur (C.G.), India

Received: 19 September 2017, Accepted: 17 November 2017

Published online: 2 May 2018.

\begin{abstract}
In this paper, a new type of generalized quasi-Einstein manifold is defined. The special cases of this manifold are Einstein manifold, quasi-Einstein manifold and nearly quasi-Einstein manifold. We have shown the existence of this new type of generalised quasi-Einstein manifold by a suitable example.
\end{abstract}

Keywords: Einstein manifold, quasi-Einstein manifold, nearly quasi-Einstein manifold, conformal curvature tensor, concircular curvature tensor, Einstein field equation.

\section{Introduction}

Let $\left(M^{n}, g\right),(n>2)$, be an n-dimensional Riemannian manifold. A Riemannian manifold is said to be an Einstein manifold if a non-zero Ricci tensor of the manifold satisfies relation

$$
R_{i j}=\frac{R}{n} g_{i j}
$$

where $R_{i j}, \mathrm{R}$ and $g_{i j}$ are Ricci tensor of type $(0,2)$, scalar curvature and Riemannian metric respectively.

If a non-zero Ricci tensor of the manifold satisfies relation

$$
R_{i j}=\alpha g_{i j}+\beta A_{i} A_{j}
$$

then, the manifold is called a quasi-Einstein manifold, where $A_{i}$ is a unit covariant vector on $U=\left\{x \in M: R_{i j} \neq \frac{R}{n} g_{i j}\right\}$ and $\alpha, \beta$ are scalars on $\mathrm{U}$. Generally an n-dimensional quasi-Einstein manifold is denoted by $(Q E)_{n}$. The quasi-Einstein manifold is also studied by U.C.De and G.C.Ghosh[13], C.Özgür and S. Sular[6] and A. A. Shaikh, D. W. Yoon and S. K. Hui[1] and [2,3,8,9]. According to U. C. De and A. K. Gazi [12], a manifold is said to be nearly quasi-Einstein manifold, if the non-zero Ricci tensor of the manifold satisfies the relation

$$
R_{i j}=\alpha g_{i j}+\beta E_{i j}
$$

where $E_{i j}$ is a symmetric tensor of type $(0,2)$.

In 1969 K. Yano [10] define a new type of curvature tensor by combining conformal curvature tensor and concircular curvature tensor. M. C. Chaki and M. L. Ghosh[11] also combined conformal curvature and concircular curvature tensor and gave an experession for a quasi-conformal curvature tensor $\mathrm{W}$ of type $(1,3)$ by

$$
W_{i j k}^{h}=-(n-2) b C_{i j k}^{h}+[a+(n-2) b] L_{i j k}^{h},
$$

\footnotetext{
*Corresponding author e-mail: brajbhushan25@gmail.com; brijeshggv75@gmail.com
} 
where a, b are arbitrary constants, not simultaneously zero and $C_{i j k}^{h}, L_{i j k}^{h}$ are conformal and concircular curvature tensor respectively.

From (4) we can say that the quasi-conformal curvature tensor will be equal to conformal curvature tensor or concircular curvature tensor according as $a=1$ and $b=-\frac{1}{n-2}$ or $a=1$ and $b=0$ respectively.

We know that the conformal and concircular curvature tensors are defined by

$$
C_{i j k}^{h}=R_{i j k}^{h}-\frac{1}{n-2}\left(R_{i j} \delta_{k}^{h}-R_{i k} \delta_{j}^{h}+R_{k}^{h} g_{j i}-R_{j}^{h} g_{k i}\right)+\frac{R}{(n-1)(n-2)}\left(g_{i j} \delta_{k}^{h}-\delta_{j}^{h} g_{k i}\right),
$$

and

$$
L_{i j k}^{h}=R_{i j k}^{h}-\frac{R}{n(n-1)}\left(g_{i j} \delta_{k}^{h}-\delta_{j}^{h} g_{k i}\right) .
$$

Putting the values of conformal and concircular curvature tensors from (5) and (6) in (4) we have an expression for quasi-conformal curvature tensor $\mathrm{W}$ of type $(1,3)$, given by

$$
W_{i j k}^{h}=a R_{i j k}^{h}+b\left(R_{i j} \delta_{k}^{h}-R_{i k} \delta_{j}^{h}+R_{k}^{h} g_{j i}-R_{j}^{h} g_{k i}\right)-\frac{R\left[\frac{a}{n-1}+2 b\right]}{n}\left(g_{i j} \delta_{k}^{h}-\delta_{j}^{h} g_{k i}\right) .
$$

\section{A new type of generalized quasi-Einstein manifold}

Now we define a manifold and called it new type of generalized quasi-Einstein manifold in which a non-zero Ricci tensor satisfies a different type of relation. Generalized in sense that special cases of this manifold are an Einstein manifold, quasi-Einstein manifold and nearly quasi-Einstein manifold.

Definition 1. Let $\left(M^{n}, g\right),(n>2)$, be a Riemannian manifold. If the non-zero Ricci tensor of the manifold satisfies the relation

$$
R_{i j}=\alpha g_{i j}+\beta A_{i} A_{j}+[\alpha-(n-2) \gamma] E_{i j},
$$

then, the manifold is called a new type of generalized quasi-Einstein manifold, where $R_{i j}, E_{i j}$ and $\alpha, \beta, \gamma$ are Ricci tensor, symmetric tensor of type $(0,2)$ and scalars respectively.

Transvecting (8) by $g^{i j}$, we have

$$
R=\alpha n+\beta+[\alpha-(n-2) \gamma] E,
$$

where $E=E_{i j} g^{i j}$ and $A^{j} A_{j}=1\left(A^{j}=A_{i} g^{i j}\right)$. From (8) three cases arise.

Case i. if $\beta=0$ and $\alpha=(n-2) \gamma$ then from (8), we get

$$
R_{i j}=(n-2) \gamma g_{i j}
$$

then, manifold becomes Einstein manifold.

Case ii. if $\alpha=(n-2) \gamma$ then from (8), we get

$$
R_{i j}=(n-2) \gamma g_{i j}+\beta A_{i} A_{j}
$$

then, manifold becomes a quasi-Einstein manifold. 
Case iii. if $\beta=0$ and $\alpha \neq(n-2) \gamma$ then from (8), we get

$$
R_{i j}=\alpha g_{i j}+[\alpha-(n-2) \gamma] E_{i j}
$$

then, manifold becomes a nearly quasi-Einstein manifold.

Now using (8) and (9) in (7), we obtain an expression for conformal curvature tensor in a new type of generalized quasi-Einstein manifold, given by

$$
\begin{aligned}
W_{i j k}^{h}= & a R_{i j k}^{h}+b \beta\left[\delta_{k}^{h} A_{i} A_{j}-\delta_{j}^{h} A_{i} A_{k}+A_{k} A^{h} g_{i j}-A_{j} A^{h} g_{i k}\right]+b[\alpha-(n-2) \gamma]\left(\delta_{k}^{h} E_{i j}-\delta_{j}^{h} E_{i k}+E_{k}^{h} g_{j i}-E_{j}^{h} g_{k i}\right) \\
& -\left[(\alpha-(n-2) \gamma)\left(\frac{a+2(n-1) b}{n(n-1)}\right) E+\frac{\beta A}{n}\left(\frac{a}{n-1}+2 b\right)+\frac{\alpha a}{(n-1)}\right]\left(g_{i j} \delta_{k}^{h}-\delta_{j}^{h} g_{k i}\right) .
\end{aligned}
$$

Now, we consider a quasi-conformally flat new type of generalized quasi-Einstein manifold i.e. $W_{i j k}^{h}=0$, then from (13), we get

$$
\begin{aligned}
R_{i j k}^{h}= & -\frac{b \beta}{a}\left[\delta_{k}^{h} A_{i} A_{j}-\delta_{j}^{h} A_{i} A_{k}+A_{k} A^{h} g_{i j}-A_{j} A^{h} g_{i k}\right]-\frac{b}{a}[\alpha-(n-2) \gamma]\left(\delta_{k}^{h} E_{i j}-\delta_{j}^{h} E_{i k}+E_{k}^{h} g_{j i}-E_{j}^{h} g_{k i}\right) \\
& +\frac{1}{a}\left[(\alpha-(n-2) \gamma)\left(\frac{a+2(n-1) b}{n(n-1)}\right) E+A \frac{\beta}{n}\left(\frac{a}{n-1}+2 b\right)+\frac{\alpha a}{(n-1)}\right]\left(g_{i j} \delta_{k}^{h}-\delta_{j}^{h} g_{k i}\right) .
\end{aligned}
$$

Equation (14) can be written as

$$
\begin{aligned}
R_{i j k}^{h}= & P\left[\delta_{k}^{h} A_{i} A_{j}-\delta_{j}^{h} A_{i} A_{k}+A_{k} A^{h} g_{i j}-A_{j} A^{h} g_{i k}\right]+Q\left(\delta_{k}^{h} E_{i j}-\delta_{j}^{h} E_{i k}\right. \\
& \left.+E_{k}^{h} g_{j i}-E_{j}^{h} g_{k i}\right)+(S E+K A+U)\left(g_{i j} \delta_{k}^{h}-\delta_{j}^{h} g_{k i}\right),
\end{aligned}
$$

where $P=-\frac{b \beta}{a}, \quad Q=-\frac{b}{a}[\alpha-(n-2) \gamma], \quad S=\frac{1}{a}[\alpha-(n-2) \gamma]\left(\frac{a+2(n-1) b}{n(n-1)}\right), \quad K=\frac{\beta}{a n}\left[\frac{a}{n-1}+2 b\right], a \neq 0$ and $U=\frac{\alpha}{(n-1)}$. Now, if we take

$$
Q\left(\delta_{k}^{h} E_{i j}-\delta_{j}^{h} E_{i k}+E_{k}^{h} g_{j i}-E_{j}^{h} g_{k i}\right)+(S E+K A)\left(g_{i j} \delta_{k}^{h}-\delta_{j}^{h} g_{k i}\right)=0,
$$

then from (16) and (15), we obtain

$$
R_{i j k}^{h}=P\left[\delta_{k}^{h} A_{i} A_{j}-\delta_{j}^{h} A_{i} A_{k}+A_{k} A^{h} g_{i j}-A_{j} A^{h} g_{i k}\right]+U\left(g_{i j} \delta_{k}^{h}-\delta_{j}^{h} g_{k i}\right) .
$$

In 1972 Chen and Yano [7] gave the concept of a manifold of quasi-constant curvature tensor and define.

Definition 2. A Riemannian manifold $\left(M^{n}, g\right),(n>3)$ is said to be a manifold of quasi-constant curvature if it is conformally flat and its curvature tensor $R_{i j k}^{h}$ of type $(1,3)$ have the form

$$
R_{i j k}^{h}=U\left[\delta_{k}^{h} A_{i} A_{j}-\delta_{j}^{h} A_{i} A_{k}+A_{k} A^{h} g_{i j}-A_{j} A^{h} g_{i k}\right]+V\left(g_{i j} \delta_{k}^{h}-\delta_{j}^{h} g_{k i}\right),
$$

where $A_{i}$ is a covariant vector and $U, V$ are scalars of which $V \neq 0$.

Thus, from (15), (16) and (17) we conclude that

Theorem 1. A quasi-conformally flat new type of generalized quasi-Einstein manifold will be a manifold of quasi-constant curvature if and only if

$$
\delta_{k}^{h} E_{i j}-\delta_{j}^{h} E_{i k}+E_{k}^{h} g_{j i}-E_{j}^{h} g_{k i}+(S E+K A)\left(g_{i j} \delta_{k}^{h}-\delta_{j}^{h} g_{k i}\right)=0 .
$$

Now, we propose. 
Corollary 1. If a quasi-conformally flat new type of generalized quasi-Einstein manifold be a manifold of quasi-constant curvature then the symmetric tensor $E_{i j}$ satisfies the relation

$$
E_{i j}=-\frac{K}{2 Q+n S} A_{i} A_{j}
$$

Proof. From Theorem 1. it is clear that if a quasi-conformally flat new type of generalized quasi-Einstein manifold be a manifold of quasi-constant curvature then

$$
Q\left(\delta_{k}^{h} E_{i j}-\delta_{j}^{h} E_{i k}+E_{k}^{h} g_{j i}-E_{j}^{h} g_{k i}\right)+(S E+K A)\left(g_{i j} \delta_{k}^{h}-\delta_{j}^{h} g_{k i}\right)=0 .
$$

Contracting in $\mathrm{h}$ and $\mathrm{k}$, we get

$$
(n-1)(2 Q+n S) E_{i j}+(n-1) K A_{i} A_{j}=0,
$$

equation (20)which implies that

$$
E_{i j}=-\frac{K}{2 Q+n S} A_{i} A_{j}
$$

Contracting (17) in h and k, we get

$$
R_{i j}=P\left[n A_{i} A_{j}-A_{i} A_{j}+A_{i} A^{i} g_{i j}-A_{j} A_{i}\right]+U\left(n g_{i j}-g_{j i}\right),
$$

equation (22)which implies that

$$
R_{i j}=(n-1) U g_{i j}+P(n-1) A_{i} A_{j} .
$$

Taking $v=[(n-1) U]$ and $\mu=[P(n-1)]$, in (23), we get

$$
R_{i j}=v g_{i j}+\mu A_{i} A_{j}
$$

This is a quasi-Einstein manifold. Thus we conclude that.

Theorem 2. If a quasi-conformally flat new type of generalized quasi-Einstein manifold be a manifold of quasi-constant curvature then this becomes a quasi-Einstein manifold.

\section{Einstein field equation in a new type of generalized quasi-Einstein manifold}

The Einstein field equation with a cosmological term is given by [8]

$$
R_{i j}-R \frac{1}{2} g_{i j}+\wedge g_{i j}=k T_{i j}
$$

where $\wedge, \mathrm{k}$ and $T_{i j}$ are cosmological constant, Gravitational constant and energy momentum tensor respectively.

Using (8) and (9) in (25), we have

$$
\alpha g_{i j}+\beta A_{i} A_{j}+[\alpha-(n-2) \gamma] E_{i j}-\frac{1}{2}(\alpha n+\beta A+[\alpha-(n-2) \gamma] E) g_{i j}+\wedge g_{i j}=k T_{i j}
$$

equation (26) which implise that

$$
\left(\alpha\left(1-\frac{n}{2}\right)+\wedge\right) g_{i j}+\beta \frac{1}{2} A_{i} A_{j}+\left(1-\frac{n}{2}\right)[\alpha-(n-2) \gamma] E_{i j}=k T_{i j}
$$


which is required Einstein field equation in a new type of generalized quasi-Einstein manifold. Taking covariant derivative of (27) and suppose $\nabla_{j} A_{i}=0$, we have

$$
\left(\alpha\left(1-\frac{n}{2}\right)+\wedge\right) \nabla_{h} g_{i j}+\left(1-\frac{n}{2}\right)[\alpha-(n-2) \gamma] \nabla_{h} E_{i j}=k \nabla_{h} T_{i j}
$$

equation (28)which implies that

$$
\left(1-\frac{n}{2}\right)[\alpha-(n-2) \gamma] \nabla_{h} E_{i j}=k \nabla_{h} T_{i j}
$$

Thus, we conclude.

Theorem 3. In a new type of generalized quasi-Einstein manifold, if $A_{i}$ be covariant constant then

(i) Symmetric tensor $E_{i j}$ is covariant constant if the energy momentum tensor is covariant constant,

(ii) Symmetric tensor $E_{i j}$ is recurrent if the energy momentum tensor is recurrent.

Taking covariant derivative of (27) and $A_{i}$ be a covariant constant, we get

$$
\left(\alpha\left(1-\frac{n}{2}\right)+\wedge\right) g_{i j, k}+\left(1-\frac{n}{2}\right)[\alpha-(n-2) \gamma] E_{i j, k}=k T_{i j, k},
$$

Interchanging $\mathrm{i}, \mathrm{j}$ and $\mathrm{k}$ in cyclic order in (30), we have

$$
\left(\alpha\left(1-\frac{n}{2}\right)+\wedge\right) g_{j k, i}+\left(1-\frac{n}{2}\right)[\alpha-(n-2) \gamma] E_{j k, i}=k T_{j k, i},
$$

and

$$
\left(\alpha\left(1-\frac{n}{2}\right)+\wedge\right) g_{k i, j}+\left(1-\frac{n}{2}\right)[\alpha-(n-2) \gamma] E_{k i, j}=k T_{k i, j} .
$$

Adding (30), (31) and (32), we get

$$
\left(1-\frac{n}{2}\right)[\alpha-(n-2) \gamma]\left(E_{i j, k}+E_{j k, i}+E_{k i, j}\right)=k\left(T_{i j, k}+T_{j k, i}+T_{k i, j}\right) .
$$

Now, if symmetric tensor $E_{i j}$ satisfies the Bianchi second identity, then

$$
E_{i j, k}+E_{j k, i}+E_{k i, j}=0
$$

therefore from (33), we get

$$
T_{i j, k}+T_{j k, i}+T_{k i, j}=0 \text {. }
$$

i.e. the energy momentum tensor satisfies the Bianchi second identity. Thus, we conclude that.

Theorem 4. In a new type of generalized quasi-Einstein manifold if $A_{i}$ be covariant constant then the symmetric tensor $E_{i j}$ satisfies the Bianchi second identity if and only if energy momentum tensor satisfies the Bianchi second identity.

\section{An example of a new type of generalized quasi-Einstein manifold}

Now, we take a manifold (M, g) such that $M=R^{4}$ and the metric $\mathrm{g}$ in $R^{4}$ is given by

$$
d s^{2}=g_{i j} d x^{i} d x^{j}=f\left(x^{4}\right)\left[\left(d x^{1}\right)^{2}+\left(d x^{2}\right)^{2}+\left(d x^{3}\right)^{2}\right]+\left(d x^{4}\right)^{2},
$$


the only non-vanishing components of christoffel symbols and the curvature tensor are given by

$$
\Gamma_{11}^{4}=\Gamma_{33}^{4}=\Gamma_{22}^{4}=-\frac{1}{2} f^{\prime}\left(x^{4}\right), \Gamma_{14}^{1}=\Gamma_{34}^{3}=\Gamma_{24}^{2}=\frac{1}{2}\left(\frac{f^{\prime}\left(x^{4}\right)}{f\left(x^{4}\right)}\right),
$$

and

$$
R_{1441}=R_{2442}=R_{4334}=\frac{1}{2} f^{\prime \prime}\left(x^{4}\right)-\frac{1}{4} \frac{\left(f^{\prime}\left(x^{4}\right)\right)^{2}}{f\left(x^{4}\right)}, R_{2112}=R_{3113}=R_{2332}=\frac{1}{4}\left(f^{\prime}\left(x^{4}\right)\right)^{2}
$$

The only non-zero Rici tensors are given by

$$
R_{11}=R_{22}=R_{33}=-\frac{1}{2} f^{\prime \prime}\left(x^{4}\right)-\frac{1}{2} \frac{\left(f^{\prime}\left(x^{4}\right)\right)^{2}}{f\left(x^{4}\right)}, \quad R_{44}=-\frac{3 f^{\prime \prime}\left(x^{4}\right)}{2 f\left(x^{4}\right)}+\frac{3}{4}\left(\frac{f^{\prime}\left(x^{4}\right)}{f\left(x^{4}\right)}\right)^{2}
$$

assuming

$$
\begin{gathered}
\alpha=-\frac{f^{\prime \prime}\left(x^{4}\right)}{2 f\left(x^{4}\right)}-\frac{1}{2}\left(\frac{f^{\prime}\left(x^{4}\right)}{f\left(x^{4}\right)}\right)^{2}, \beta=\frac{3}{2 f\left(x^{4}\right)}\left\{\frac{f^{\prime \prime}\left(x^{4}\right)}{f\left(x^{4}\right)}-\left(\frac{f^{\prime}\left(x^{4}\right)}{f\left(x^{4}\right)}\right)^{2}\right\}, \gamma=-\left(\frac{f^{\prime}\left(x^{4}\right)}{f\left(x^{4}\right)}\right)^{2}+\frac{1}{2} \frac{f^{\prime \prime}\left(x^{4}\right)}{f\left(x^{4}\right)}, \\
A_{i}= \begin{cases}\sqrt{f\left(x^{4}\right)}, & i=1 \\
0, & i=2,3,4,\end{cases}
\end{gathered}
$$

and

$$
E_{i j}= \begin{cases}1, & i=j=1 \\ 0, & i \neq j \text { and } i=j=2,3 \\ \frac{1}{6}\left(\frac{4 f^{\prime \prime}\left(x^{4}\right) f\left(x^{4}\right)-5 f^{\prime}\left(x^{4}\right)^{2}}{f^{\prime \prime}\left(x^{4}\right) f\left(x^{4}\right)-f^{\prime}\left(x^{4}\right)^{2}}\right), & i=j=4 .\end{cases}
$$

Now, using above value, we have

$$
\begin{aligned}
& \alpha g_{11}+\beta A_{1} A_{1}+(\alpha-2 \gamma) E_{11}=-\frac{1}{2} f^{\prime \prime}\left(x^{4}\right)-\frac{1}{2} \frac{\left(f^{\prime}\left(x^{4}\right)\right)^{2}}{f\left(x^{4}\right)}, \\
& \alpha g_{22}+\beta A_{2} A_{2}+(\alpha-2 \gamma) E_{22}=-\frac{1}{2} f^{\prime \prime}\left(x^{4}\right)-\frac{1}{2} \frac{\left(f^{\prime}\left(x^{4}\right)\right)^{2}}{f\left(x^{4}\right)}, \\
& \alpha g_{33}+\beta A_{3} A_{3}+(\alpha-2 \gamma) E_{33}=-\frac{1}{2} f^{\prime \prime}\left(x^{4}\right)-\frac{1}{2} \frac{\left(f^{\prime}\left(x^{4}\right)\right)^{2}}{f\left(x^{4}\right)}, \\
& \alpha g_{44}+\beta A_{4} A_{4}+(\alpha-2 \gamma) E_{44}=-\frac{3 f^{\prime \prime}\left(x^{4}\right)}{2 f\left(x^{4}\right)}+\frac{3}{4}\left(\frac{f^{\prime}\left(x^{4}\right)}{f\left(x^{4}\right)}\right)^{2} .
\end{aligned}
$$

Now, from equation (39) and (40), we have

(1) $R_{11}=\alpha g_{11}+\beta A_{1} A_{1}+(\alpha-2 \gamma) E_{11}$,

(2) $R_{22}=\alpha g_{22}+\beta A_{2} A_{2}+(\alpha-2 \gamma) E_{22}$,

(3) $R_{33}=\alpha g_{33}+\beta A_{3} A_{3}+(\alpha-2 \gamma) E_{33}$,

(4) $R_{44}=\alpha g_{44}+\beta A_{4} A_{4}+(\alpha-2 \gamma) E_{44}$.

We shall now show that the 1 -forms are unit

$$
g^{i j} A_{i} A_{j}=g^{11} A_{1} A_{1}+g^{22} A_{2} A_{2}+g^{33} A_{3} A_{3}+g^{44} A_{4} A_{4}=1,
$$


this shows that $\left(R^{4}, g\right)$ is a new type of generalized quasi-Einstein manifold.

\section{Acknowledgements}

The second author express his thanks to (UGC) New Delhi, India for providing Junior Research Fellowship (JRF).

\section{Competing interests}

The authors declare that they have no competing interests.

\section{Authors' contributions}

All authors have contributed to all parts of the article. All authors read and approved the final manuscript.

\section{References}

[1] A. A. Shaikh D. W. Yoon and S. K. Hui, On quasi-Einstein spacetimes, Tsukuba J. Math. 33, 2, (2009), 305-306.

[2] A. A. Shaikh, Y. H. Kim and S. K. Hui, On Lorentzian quasi-Einstein manifolds, J. Korean Math. Soc., 48(4), (2011), 669-689.

[3] A. A. Shaikh and S. K. Hui, On decomposable quasi-Einstein spaces, Math, Reports, 13(63)(2011), 89-94.

[4] A. A. Shaikh and A. Patra, On quasi-conformally flat quasi-Einstein spaces, Differ. Geom. Dyn. Syst. 12, (2010), 201-212.

[5] B. Y. Chen and K.Yano, Hypersurfaces of a conformally flat space, Tensor N.S. 26, (1972), 318-322.

[6] C.Özgür and S. Sular, On N(k)- quasi-Einstein manifold satisfies certain condition, Balkan J. Geom. and Its Appl. 13, 2, (2008), 74-79.

[7] S. W. Hawking and G.F.R. Ellis, The large scale structure of space-time, Cambridge University Press, Cambridge, 1973.

[8] S. K. Hui, A note on nearly quasi-Einstein manifolds, Europe J. Pure Applied Math. 5(3) (2012), 365-372.

[9] S. K. Hui R. S. Lemence and M. P. Roque, Sectional curvatures of quasi-Einstein manifolds, Int. J. Math. Analysis, 7(2013), 2833-2844.

[10] K. Yano, Riemannian manifolds admitting a conformal transformation group, Proc. Natl Acad Sci. USA, 62, 2, (1969), $314-319$.

[11] M. C. Chaki and M. L. Ghosh, On quasi conformally flat and quasi conformally conservative Riemannian manifolds, Analele Stiint. Ale Universitatee"AL. I. Cuza” Iasi, Tomul XLIII, Mathematica, 1997, 375-381.

[12] U.C.De and A. K. Gazi, On nearly quasi Einstein manifolds, Novi Sad J. Math. 38, 2, (2008), 115-121.

[13] U.C.De and G.C.Ghosh, On quasi-Einstein manifolds,Period. Math. Hung. 48, (1-2), (2004), 223-231. 